POS PROCEEDINGS

\title{
Recent progress of the Baikal-GVD project
}

\author{
A.D. Avrorin ${ }^{a}$, A.V. Avrorin ${ }^{a}$, V.M. Aynutdinov ${ }^{a}$, R. Bannash ${ }^{g}$, I.A Belolaptikov ${ }^{b}$, \\ V.B. Brudanin ${ }^{b}$, N.M. Budnev ${ }^{c}$, G.V. Domogatsky ${ }^{a}$, A.A. Doroshenko ${ }^{a}$, \\ R. Dvornický ${ }^{b, h}$, A.N. Dyachok ${ }^{c}$, Zh.-A.M. Dzhilkibaev ${ }^{a}$, L. Fajt ${ }^{b, h, i}$, S.V Fialkovsky ${ }^{e}$, \\ A.R. Gafarov ${ }^{c}$, K.V. Golubkov ${ }^{a}$, N.S. Gorshkov ${ }^{b}$, T.I. Gress ${ }^{c}$, R. Ivanov ${ }^{b}$, \\ K.G. Kebkal ${ }^{g}$, O.G. Kebkal ${ }^{g}$, E.V. Khramov ${ }^{b}$, M.M. Kolbin ${ }^{b}$, K.V. Konischev ${ }^{b}$, \\ A.V. Korobchenko ${ }^{b}$, A.P. Koshechkin ${ }^{a}$, A.V. Kozhin ${ }^{d}$, M.V. Kruglov ${ }^{b}$, M.K. Kryukov ${ }^{a}$, \\ V.F. Kulepov ${ }^{e}$, M.B. Milenin ${ }^{a}$, R.A. Mirgazov ${ }^{c}$, V. Nazari ${ }^{b}$, A.I. Panfilov $^{a}$, \\ D.P. Petukhov ${ }^{a}$ E.N. Pliskovsky ${ }^{b}$, M.I. Rozanov ${ }^{f}$, E.V. Rjabov ${ }^{c}$, V.D. Rushay ${ }^{b}$, \\ G.B. Safronov ${ }^{b}$, B.A. Shaybonov ${ }^{b}$, M.D. Shelepov ${ }^{a}$, F. Šimkovic ${ }^{b, h, i}$, A.V. Skurikhin ${ }^{d}$, \\ A.G. Solovjev $^{b}$, M.N. Sorokovikov ${ }^{b}$, I. S̆tekI $^{i}$, E.O. Sushenok $^{b}$, O.V. Suvorova ${ }^{* a}$, \\ V.A. Tabolenko ${ }^{c}$, B.A. Tarashansky ${ }^{c}$, and S.A. Yakovlev ${ }^{g}$ \\ a Institute for Nuclear Research, Russian Academy of Sciences, Moscow, 117312 Russia \\ ${ }^{b}$ Joint Institute for Nuclear Research, Dubna, 141980 Russia \\ ${ }^{c}$ Irkutsk State University, Irkutsk, 664003 Russia \\ ${ }^{d}$ Institute of Nuclear Physics, Moscow State University, Moscow, 119991 Russia \\ e Nizhni Novgorod State Technical University, Nizhni Novgorod, 603950 Russia \\ ${ }^{f}$ St. Petersburg State Marine Technical University, St. Petersburg, 190008 Russia \\ ${ }^{g}$ EvoLogics Gmbh, Germany \\ ${ }^{h}$ Comenius University, Mlynska Dolina F1, Bratislava, 84248 Slovakia \\ ${ }^{i}$ Czech Technical University in Prague, Prague, 12800 Czech Republic \\ E-mail: suvorova@inr.ru
}

Cubic kilometer scale neutrino telescope Baikal-GVD is currently under construction in Lake Baikal. The detector is specially designed for search for high energies neutrinos whose sources are not yet reliably identified. Since April 2019 the telescope has been successfully operated in complex of five functionally independent clusters i.e. sub-arrays of optical modules where now are hosted 1440 OMs on 40 vertical strings. Each cluster is connected to shore by individual electro-optical cables. The effective volume of the detector for neutrino initiated cascades of relativistic particles with energy above $100 \mathrm{TeV}$ has been increased up to about $0.25 \mathrm{~km}^{3}$. Preliminary results with data samples of 2015-2018 years are discussed.

European Physical Society Conference on High Energy Physics - EPS-HEP2019 -

10-17 July, 2019

Ghent, Belgium

${ }^{*}$ Speaker. 


\section{Introduction}

In the season of this year 2019 the Baikal-GVD Collaboration is reached a significant progress in constructing neutrino detector of gigaton volume. Since April 2019 the GVD detector successfully operated in complex of 5 functionally independent clusters of optical modules (OMs). Total effective volume is up to about $0.25 \mathrm{~km}^{3}$ for detection of neutrinos initiating cascades of relativistic particles with energy above $100 \mathrm{TeV}$. That is a quater of the IceCube detector volume in the South Pole and the largest sizes of operating neutrino telescope in the Northern hemisphere. In timeline in two nearest years (2020-2021) the first phase of the GVD construction is aimed on reaching an effective volume as large as $0.4 \mathrm{~km}^{3}$. Construction and continuous operation of gigaton volume neutrino telescopes viewed both sky hemispheres is highly relevant for acquiring complementary astrophysical neutrino observations for fluences identified by the IceCube detector since 2013 [1]. That is evidence for the first observed $\mathrm{PeV}$-scale events of unidentified origins and the first neutrino alert event defined as the neutrino fluence of $290 \mathrm{TeV}$ from BL LAC galaxy TX-0506-056 (event IC170922A [2]). Nowadays combined analysis in astronomy extended to multi-messenger astronomy. So that the combined analysis of data acquired at multiple wavelengths provides a better understanding of physical processes of particles production with energies above hundreds of $\mathrm{TeV}$ and $\mathrm{PeV}$ inside astrophysical sources and allows us to discover new sources of this kind. The Baikal-GVD following up alerts from other telescopes, involved in the multi-messenger program.

The neutrino telescope Baikal-GVD is deployed in the southern part of Lake Baikal [3], about four kilometres away from the shore, with coordinates: $104.33^{\circ}$ East longtitude and $51.83^{\circ}$ North latitude. The Lake depth at the site is $\sim 1360 \mathrm{~m}$. The site was chosen because of an optimal combination of hydrological, hydro-physical and landscape factors for deployment and operation of a neutrino telescope. Baikal-GVD has a modular structure and consists of functionally independent sub-arrays (clusters). Each cluster is an array of photodetectors (optical modules, OMs), i.e. photomultiplier tubes housed in transparent pressure spheres [4]. In a baseline cluster configuration there are 288 OMs installed on 8 vertical load-bearing cables (strings) with instrumented length of 525 meters (active depths $735-1260 \mathrm{~m}$ ). Each cluster is connected to the shore via an individual electro-optical cable used for transmitting electrical power and data.

The neutrino telescope has been in permanent operation since April 2015, when the first GVD cluster was commissioned. Each year new cluster (or two) is deployed and, starting April 2019, five clusters of the telescope totalling $1440 \mathrm{OMs}, 120$ sections and 40 strings have been acquiring data. In this contribution we shortly review the detector performance and some preliminary results of data analysis Ref. [5].

\section{The GVD basic structures}

The first configuration of the Baikal-GVD was the demonstration cluster "Dubna" [6] consisted of eight $345 \mathrm{~m}$ long strings comprised two sections of OMs of optical modules, spaced at $40 \mathrm{~m}$ (192 OMs in total). After successful operation of "Dubna" in season April 2015 - February 2016, in next winter expedition 2016 the cluster has been extended to the baseline configuration of GVD cluster by additional third top sections at each string and by prolongation of radius to 60 meters for 7 side strings from central one. The upgraded cluster comprises 288 OMs operated at active depths of 



Figure 1: Left: Schematic drawing of the Baikal-GVD five clusters operated in 2019. Right: Integrated number of events recorded by the clusters in period of April-July of 2019. Different colors mark clusters.

735-1260 meters. Instrumented volume of single cluster encloses 5.9 Megatons. During 2017 and 2018, each year, one new cluster had been deployed and two new clusters deployed in April 2019. The cluster centers are spaced at 300 meters. Currently, five clusters are operated, as shown in Fig. 1 (left) schematically. The start year of each cluster is also indicated.

Basic element of the Baikal-GVD structure is a section of 12 OMs distributed vertically along the string, spaced by $15 \mathrm{~m}$, and a central electronics module (CeM). There are 3 sections of $36 \mathrm{OMs}$ per string and 8 strings in cluster, each is an independently working sub-array of the Baikal-GVD. One photomultiplier tube Hamamatsu R7081-100 with a 10-inch hemispherical photocathode and quantum efficiency up to $35 \%$ is used as light sensor of OM [7]. The signals arriving from the OMs are digitized in ADC boards located at the CeM (12 channels with sampling frequency $200 \mathrm{MHz}$ ). When digitized, the signal from the ADC is transferred into programmable gate array (FPGA), which logic has been configured for generating a trigger, reading data from the ADC channels, and transmitting them over the Ethernet channel into the data acquisition center. A basic trigger mode is a signal amplitude in 4 FADC counts exceeding low and high thresholds in two adjacent OMs within $100 \mathrm{~ns}$ in a section: for thresholds 1.5 p.e. and 4.5 p.e. the trigger rate is in range of $60-110$ $\mathrm{Hz}$. When the trigger condition is fulfilled, a request signal is generated to the cluster center module (CC) through $1 \mathrm{~km}$ long line. CC generates an acknowledgement signal to all CMs in a cluster, when the request signal is received. When CM is caught the signal, a timestamp is defined and the $\mathrm{CM}$ starts to form data. The FPGA memory buffer allows to acquire the OM signal waveform for a time interval as long as $5 \mu \mathrm{s}$, this information then is transferred to the shore station and stored as raw data in binary files. Such a trigger system approach allows to have all signal waveforms on each channel in an event from only one triggered pair of adjacent channels, while the data arriving from the sections can be processed in real time mode. Additionally, there are raw data from the acoustic positioning system and from OM monitoring. Details of data acquisition, basic controls, methods of calibrations, hard-and soft-ware triggers can be found in [8] and [9], as well the monitoring of water noise in [10] and the data quality monitoring in [11]. The cumulative number of section records generated by each array in period of April-July of 2019 are shown in Fig. 1 (right). 


\section{Seacrh for high energy neutrinos}

Data analysis is based on two modes in the reconstruction of Cherenkov radiation being emitted either by cascade particles or by straight-line moving muons generated in the HE neutrino interactions with Baikal water Ref. [12]. Rates of noise impulses vary between 20 and $100 \mathrm{kHz}$ depending on season and depth, and this is suppressed by a causality requirement $\pm 10 \mathrm{~ns}$. Two typical plots for track-like and cascade-like reconstructed events are shown in Fig. 2.
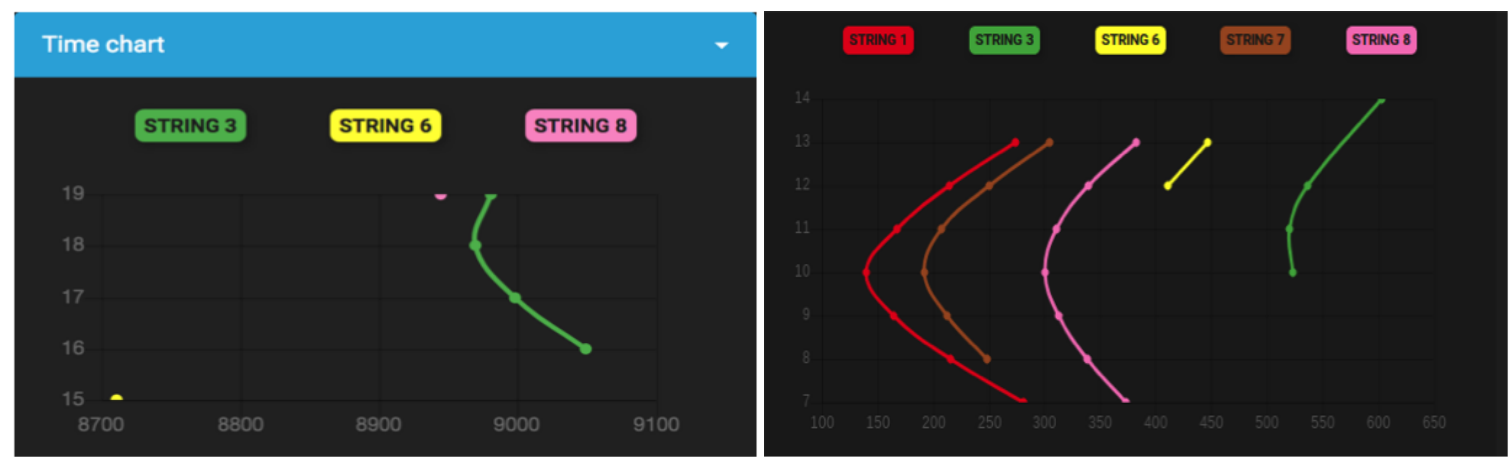

Figure 2: Z-coordinates of hit OMs versus arrival time of a Cherenkov photons. Left: Typical reconstruction of a muon track-like event. Right: Typical reconstructed cascade-like event.

In the cascade mode the accuracy of direction reconstruction is about $4.5^{\circ}$ (median value). The energy resolution averaged by an $\mathrm{E}^{-2}$ spectrum of electron neutrinos is about $30 \%$, about $90 \%$ of these events are within range of $5 \mathrm{TeV}<E<10 \mathrm{PeV}$. Shown in Fig. 3 (left) are distributions of the expected number of events per year from astrophysical flux with $E^{-2.46}$ spectrum and the IceCube normalization and from atmospheric muons. An estimate of the number of astrophysical events of an $\mathrm{E}^{-2.46}$ spectrum with energies above $100 \mathrm{TeV}$ is about 0.6 per cluster per year and about 0.08 for background events. Applied quality cuts reject events by number of hits on a string $(>6)$, by the value of $\chi^{2}$ in the reconstruction of the vertex position and by the likelihood value in the reconstruction of energy and direction and as well by the product of probabilities of hits and no hits across correspondent optical modules. In the trajectory mode a procedure based on BDT (in TMVA implementation) was applied. The obtained angular resolution is about $1^{\circ}$ as median value, while the energy release is not yet estimated. Totally, for further progress in track reconstraction new methods are incorporated for surviving true hits upto $94 \%$.

The first neutrino candidate with energy $107 \mathrm{TeV}$ have been found with data sample of 2015 of the cluster "Dubna", found coordinates are $\delta=5.56^{\circ}, \alpha=139.7^{\circ}$ for MJD 57342. [6]. Presently, to search for high energy neutrinos of astrophysical origin the data collected by one cluster in 2016 and by three clusters in 2018 have been used. A data sample of $3.8 \times 10^{9}$ events has been accumulated by the array trigger, which corresponds to 872 one cluster live days ( 2.4 years $\times$ cluster). After applying procedures of cascade vertex and energy reconstruction for hits with charge higher than 1.5 ph.el., 417 cascade-like events with OMs hit multiplicity $N_{\text {hit }}>13$ have been selected, their reconstructed energy is above $1 \mathrm{TeV}$. The requirement of high multiplicity allows substantial suppression of background events from atmospheric muon bundles. Application of cut on $N_{h i t}>20$ rejects statistics down to 18 events. As seen from Fig. 3 (center) where are shown cumulative energy distributions of these 18 events (black histogram) and events expected from IceCube neutrino 

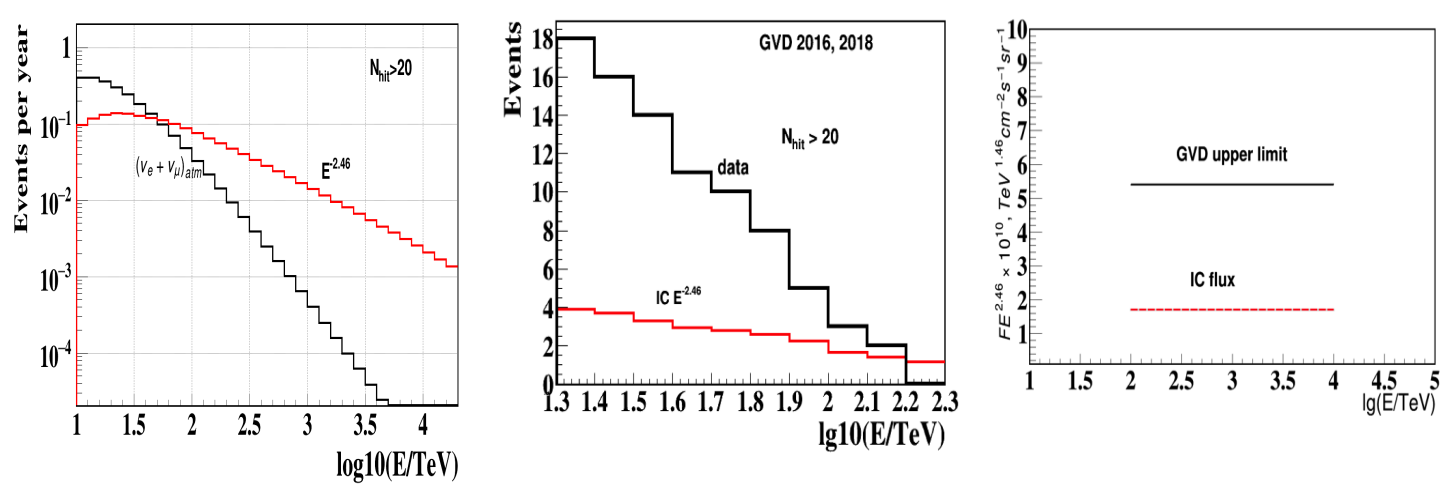

Figure 3: Left: Energy distributions of events expected for one year observation from astrophysical fluxes with $\mathrm{E}^{-2.46}$ spectra and IceCube normalization, and from atmospheric neutrinos. Center: Cumulative energy distributions of experimental events (black) and events expected from IceCube neutrino flux of astrophysical origin (red). Right: $90 \%$ CL upper limit on a single-flavor diffuse neutrino flux with $\mathrm{E}^{-2.46}$ spectrum and flux observed by IceCube.

flux of astrophysical origin (red histogram), below $100 \mathrm{TeV}$ the data are dominated by atmospheric background muons. Only 3 events are observed with energy above $100 \mathrm{TeV}$. Thus larger statistics is required for conclusion about astrophysical neutrino flux, while upper limits on diffuse singleflavor flux has been derived, as shown in Fig. 3 (right). Here we take into account the tree recorded events and 1.4 expected events from astrophysical flux. Our limits are consistent with the IceCube results. For further analysis we are considering 6 events as candidates on astrophysical origin.

\section{First results in frame of multimessenger search}

Two famous interesting sources of variable luminosity of multimessenger interest are the galaxy NGC 4993 and BL LAC BL LAC galaxy TX-0506+056. The first event is binary neutron stars merged and produced a gravitational wave GW170817, that was registrated by the Advanced LIGO and Advanced Virgo observatories [13]. Gamma-rays telescopes FERMI-GMB and INTEGRAL detected a short GRB (GRB170817A), associated with GW170817 in $10 \mathrm{~s}$ after and optical observations allowed the precise localization of the merger in the galaxy NGC 4993 at a distance $\sim 40 \mathrm{Mpc}$. High energy neutrino signal associated with the merger was searched for by all operating neutrino and cosmic rays telescopes. The Baikal-GVD off-line analysis has been done in the cascade mode to search for neutrino fluence associated with prompt (time window $\pm 500 \mathrm{~s}$ ) and extended gamma-rays emission (14-days time window). Our full analysis is presented in Ref. [14]. The preliminary upper limits at 90\% CL on the neutrino fluence from GW170817 for each energy decade have been derived and shown in Fig. 4 (left). The second cosmic event is the IceCube triggered neutrino alert, which is following up by multi-messenger detectors without confirmation up to now, while arising activity of BL LAC galaxy TX-0506+056 has been observed. As well, offline analysis of the Baikal-GVD showed no neutrino events associated with IC170922A have been recorded after all cuts in taken angular windows of \pm 1 hour and \pm 1 day inside $45^{\circ}<\theta<126^{\circ}$ as for the GVD site, as seen on angular distance around the source in Fig. 4 (center). 

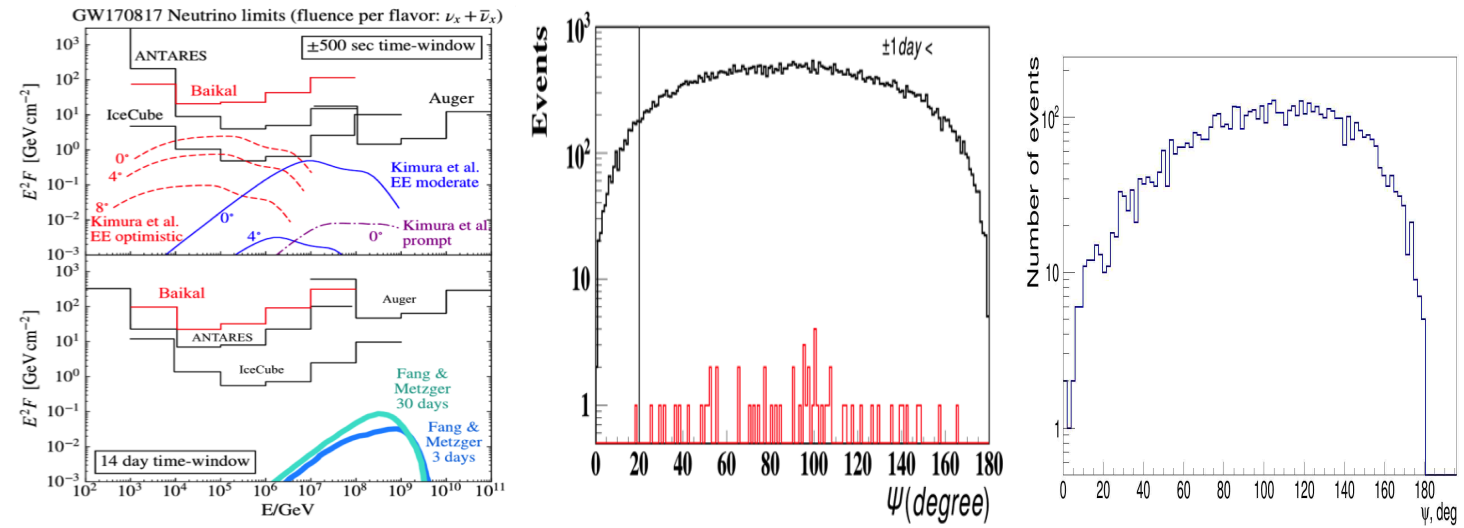

Figure 4: Left: Upper limits at 90\% C.L. on the fluence of neutrinos associated with GW170817 for prompt and delayed emission time. Center: Distribution of number of events in a time window of \pm 1 day around IC170922A directed to the blazar TX-0506+056: with only $N_{\text {hit }}$ cut (black) and with all cuts (red). Right: Mismatch angle distribution of reconstructed cascades with all cuts selection towards given ANTARES alert direction observed in 16 tested days with 3 clusters during 6 months.

The alert system of the Baikal-GVD detector is under construction to allow for a fast, on-line reconstruction of neutrino events recorded by the Baikal-GVD telescope and - if predefined conditions are satisfied - for the formation of an alert message to other communities. Upto now all raw data, for an amount as large as 40 Gbyte per cluster per day, are transferred to the shore station, stored there, and then transmitted at $5 \mathrm{Mbit} / \mathrm{s}$ on a $40 \mathrm{~km}$ channel to Baikalsk (Irkutsk region), where data are archived and transferred through a high-bandwidth internet to the central storage and processing facility in the JINR in Dubna (Moscow region) every 4 hours. New additional equipments are developed for on-line reconstruction on the shore.

Since December 2018 we involved in follow-up the ANTARES trigger. We are searching for correlations in time and direction with HE neutrino alert. We look for events per cluster in time windows of $\pm 500 \mathrm{sec}, \pm 1$ hour and \pm 1 day around alerts inside a half-open cone of $10^{\circ}$ and search for coincidence of two or more clusters within $6 \mu$ s for the first $\pm 10 \mathrm{sec}$ and in an extended interval of \pm 1 hour around the trigger. As a preliminary result, no time-direction correlations with ANTARES alerts have been found during 6 months of observations. In best case of happened coincidence within time window of \pm 1 day it is shown a distribution of values of mismatch angle with one of the ANTARES sources in Fig. 3 (center), relatively which there have been found 3 cascade events on 2 clusters among 3 of them inside of half-open cone $5^{\circ}$. An estimate of atmospheric background has been done on full statistics of reconstructed cascades for 6 months observation towards a given direction and it found to be about 1.75 events for 3 clusters per day for $\psi<10^{\circ}$ and 0.5 events for $\psi<5^{\circ}$. Further off-line analysis will follow.

In summary, the Baikal-GVD achieved important progress in the construction of telescope, it comprises total of 1440 OMs arranged at 40 strings and 5 clusters. The first few promising highenergy cascade events have been selected as candidates for events from astrophysical neutrinos with data sample of 2015-2018 years. As preliminary result, the 90\% CL upper limits on diffuse neutrino flux has been derived, which consists with current IceCube results. Following up studies has been done in frame of multi-messenger programm. 
This work was supported by the Russian Foundation for Basic Research (Grants 16-29-13032, 17-02-01237). O.V.Suvorova acknowledges support by RSCF grant 17-12-01547 in part of works in frame of multi-messenger program.

\section{References}

[1] M.G.Aartsen et al. , Phys. Rev. Lett. 113 101101. Science 3421242856 (2013).

[2] M.G.Aartsen et al., IceCube Coll., Science, 361, eaat1378 (2018)

[3] V.Aynutdinov et al., NIM A742 82-88 (2014).

[4] A.D.Avrorin et al., Phys.Part.Nucl. 46 no.2, 211-221 (2015).

[5] A.Avrorin et.al., PoS (ICRC2019)1011, (2019).

[6] A.D.Avrorin et al., EPJ Web Conf. EPJ Web Conf. 136 (2017) 04007.

[7] A.D.Avrorin et al., EPJ Web Conf. 116, 01003 (2016).

[8] A.V.Avrorin et al., Instr. and Exp. Tech. 57, issue 3, 262-273 (2014).

[9] A.Avrorin et.al., PoS (ICRC2019)1046, (2017).

[10] A.Avrorin et.al., PoS (ICRC2019)875, (2019).

[11] A.Avrorin et.al., PoS (ICRC2019)874, (2019).

[12] A.D.Avrorin et al., EPJ Web Conf. 191 (2018) 01006 (2018).

[13] B. Abbott et al., Phys. Rev. Lett., 119, 161101 (2017).

[14] A.D. Avrorin et al., arXiv:1810.10966[astro-ph.HE] submitted to Pis'ma v ZhETF. 